\title{
Calidad de sueño, síntomas depresivos y de ansiedad durante el confinamiento por COVID-I 9 en Argentina
}

\author{
Sleep quality, depressive symptoms and generalized anxiety disorders during confinement \\ by COVID-19
}

\author{
Stella M.Valiensi',Agustín L. Folgueira², Arturo D. C. Garay³
}

\section{Resumen}

En Argentina el confinamiento por la pandemia ocasionada por COVID-I 9 produjo muchos cambios. Objetivo: evaluar calidad de sueño, trastornos de ansiedad, y depresión en personas adultas. Sujetos y métodos: mediante una encuesta anónima distribuida en el país a través de la web que fue completada por 2594 personas (69\% mujeres, $32 \%$ hombres). El 30\% refirió trabajar en el ámbito de la salud. Se analizó información demográfica, calidad y otras variables de sueño, síntomas depresivos y ansiedad. Resultados: la prevalencia de malos dormidores, síntomas depresivos y ansiedad fueron de 53\%, 21,1\% y 43,8\% respectivamente. Los mayores de 65 años. mostraron una predilección mayor en acostarse antes y menor latencia de inicio de sueño. La regresión logística multivariada mostró que la edad $<55$ años y ser mujer se asoció a ansiedad y ser mal dormidor. Dormir más de 10 horas, acostarse más tarde, ser mal dormidor y ansiedad, se asoció con síntomas depresivos. Ser trabajador sanitario se asoció con tener mayor ansiedad. Conclusiones: identificamos prevalencia en mala calidad de sueño, ansiedad y depresión durante la pandemia. Más de la mitad de la población evaluada resultaron ser malos dormidores y presentaron puntajes elevados de síntomas relacionados con ansiedad y depresión. Adicionalmente los trabajadores sanitarios presentaron mayor ansiedad que el resto.

Palabras claves: COVID-19 y sueño - sueño - ansiedad - depresión.

\begin{abstract}
The confinement due to the pandemic caused by COVID-19 in Argentina produced many changes. Objective: To assess sleep, anxiety disorders, and depression in adults. Subjects and Methods: Through an anonymous survey distributed in the country through the web that was completed by 2,594 people (69\% women, $32 \%$ men). $30 \%$ reported working in the health field. Demographic information, quality and other sleep variables, depressive symptoms and anxiety were analyzed. Results: The general prevalence of bad sleepers, depressive symptoms, and anxiety were $53 \%, 21.1 \%$, and $43.8 \%$, respectively. Those over 65 years of age showed a significantly higher prevalence of going to bed earlier and having a lower sleep onset latency. Multivariate logistic regression showed that age $<55$ years and being a woman were associated with anxiety and with being a poor sleeper. Sleeping more than 10 hours, going to bed later, being a poor sleeper and anxiety, were associated with depressive symptoms. Being a healthcare worker was associated with more anxiety. Conclusions: We identified poor sleep quality and alteration mental health in times of confinement. We found that more than half of the evaluated population turned out to be poor sleepers and presented high scores of symptoms related to anxiety and depression. Additionally, health workers presented more anxiety than the rest.
\end{abstract}

Keywords: COVID-19 and sleep - sleep - anxiety - depressive symptoms.

\footnotetext{
RECIBIDO I I// I/2020 - ACEPTADO I4/I/2021

1. Médica Neuróloga,Asociación Argentina de Medicina del sueño.

2.Médico Neurólogo.Asociación Argentina de Medicina del sueño.

3.Médico Neurólogo.Asociación Argentina de Medicina del sueño. 


\section{Introducción}

La pandemia por COVID-19 ha producido una crisis en una innumerable cantidad de aspectos, en particular en lo relacionado con los efectos del aislamiento en sí mismo, en la interacción social, en lo económico, en el sueño, en la salud mental y otros fenómenos adaptativos a la inédita situación que estamos padeciendo.

A nivel social hubo que adaptarse a cambios en los sincronizadores sociales de ritmos biológicos como horarios de trabajo y escolarización online para los niños. Adaptarse a sincronizadores ambientales como ser: poca iluminación natural durante el día, disminución del ruido ambiental, exceso de uso de luz artificial (ALAN: Artificial Light at Night) (Kevin et al., 2017) por uso de celulares, participación de videos interactivos, mayor tiempo de trabajo en computadora, lo cual provoca que las personas se acuesten y se levanten más tarde de lo habitual y atrasen el inicio del sueño.

La pandemia provoca preocupaciones a nivel económico, asociadas a miedo y angustia ante la pérdida de empleo, la pérdida de ingresos, el uso de ahorros, el incremento de deudas, sobrecarga laboral. Pero también existe miedo a la enfermedad, al contagio de y por familiares, más aún en adultos mayores, también en personas con trastornos comórbidos subyacentes (Dong, y Boueym, 2020) y en trabajadores de la salud en los que el agotamiento físico, los trastornos emocionales y del sueño (Ho et al., 2020; Pappa et al., 2020) son frecuentes.

Se sabe que dormir bien, mejora el funcionamiento cerebral, el aprendizaje, ayuda a adaptarse mejor al trabajo, mejora el estado de ánimo, controla el estrés, la depresión, la ansiedad e interviene en el mantenimiento de diversas funciones metabólicas e inmunológicas, por lo que es importante tener una buena calidad de sueño durante el aislamiento que impone la pandemia.

A medida que las estadísticas de mortalidad y morbilidad alcanzan nuevos pico todos los días, el confinamiento se prolonga, las oportunidades recreativas para las personas disminuyen y la crisis financiera aumenta (Saxena, 2020), lo que lleva aparejado que las quejas por trastornos del sueño y de salud mental crezcan exponencialmente.

\section{Objetivo}

Evaluar calidad y cambios en el sueño, trastornos de ansiedad, y depresión en personas adultas durante el confinamiento por pandemia ocasionada por COVID-19.

\section{Sujetos y métodos}

Se realizó un estudio de corte transversal mediante una encuesta en forma anónima, difundida a través de las redes sociales, redes de contactos de los investigadores que integran la Asociación Argentina de Medicina del Sueño, y redes conexas inherentes a sus miembros. Este estudio se realizó de conformidad con la Declaración de Helsinki.

El consentimiento informado electrónico de querer participar, se obtuvo de cada participante antes para comenzar la investigación. El participante podía retirarse de la encuesta en cualquier momento sin proporcionar ninguna justificación.

Se utilizaron cuestionarios para medir cambios en el sueño, en la calidad del mismo, se evaluó depresión y ansiedad en sujetos mayores de 18 años que residían en Argentina en el momento del Aislamiento Social Preventivo y Obligatorio (ASPO) decretado por las autoridades.

La encuesta se aplicó entre el 13 al 30 de abril 2020, casi un mes después de implementarse el confinamiento en nuestro país. Se realizó una descripción socio-demográfica de la muestra, condiciones de trabajo y horas de exposición a COVID-19 en los que trabajan en salud. Para valorar sueño utilizamos los siguientes recursos:

a. El Índice de calidad de sueño de Pittsburgh (del inglés: Pittsburgh Sleep Quality Index, PSQI), que evalúa la calidad subjetiva, latencia del sueño, duración del sueño, eficiencia de sueño, perturbaciones del sueño, uso de medicación hipnótica y disfunción diurna. Una puntuación mayor a 5 categoriza a los participantes como malos dormidores. Puntuación de 5 o menos, son considerados buenos dormidores (Buysse et al., 1989). Una puntuación de 5 a 7 es una indicación de tratamiento médico; una puntuación de 8 a 14 implica que requiere atención y tratamiento médico y una puntuación de 14 a 21 sugiere un problema grave del sueño (Escobar-Córdoba, y Eslava-Schmalbach, 2005; Jimenez-Genchi, y Monteverde-Maldonado, 2008).

b. La Escala analógica visual para valorar calidad subjetiva de sueño, siendo 0 la peor y 10 la mejor.

c. La Escala analógica visual para valorar somnolencia diurna, siendo 0 la mínima y 10 la máxima.

d. El hábito de dormir la siesta lo evaluamos con preguntas sobre presencia (SI/NO), frecuencia (> o $<3 / 7$ días), tiempo de duración en cuarentena (igual/menor/mayor) a lo habitual.

e. Para evaluar depresión utilizamos el Cuestionario sobre la Salud del paciente-9 (PHQ-9) (Kroenke et al., 2001), que detecta la presencia, severidad de depresión y sugerencia de tratamiento. Las puntuaciones según DSMIV van de 0 a 27 puntos. 0-4: nivel de depresión negativo. 5-9: diagnóstico 
dudoso repetir el PHQ9 en el seguimiento. 10-14: moderado, se sugiere hace plan terapéutico, asesoría, seguimiento y/o recetar medicamentos.15-19: moderado-grave, se debe prescribir medicamentos con asesoramiento. 20-27: grave, prescribir medicamentos y si no hay respuesta o es deficiente, derivar a salud mental. Con adecuada sensibilidad y especificidad (88\%) para diagnóstico de depresión (Kroenke, y Spitzer, 2002; Pfizer Inc., 2010).

f. Para detectar síntomas de ansiedad utilizamos Cuestionario de Detección de Ansiedad Generalizada: GAD-7 (García-Campayo et al., 2010; Spitzer et al., 2006). Interpretación: 0-4 puntos: no se aprecian síntomas.5-10 puntos: síntomas leves. 11-15 puntos: síntomas moderados. 16-21 puntos: síntomas severos.

\section{Tamaño de la muestra}

Para el cálculo del tamaño de la muestra para la regresión logística se determinó que cada la variable dependiente (mal dormidor) tenga al menos un agregado de 10 eventos por cada una de las 12 variables independientes incluidas en el modelo. Se calculó que se obtendría un poder estadístico aceptable de este estudio si el tamaño de la muestra fuera mayor que 302 sujetos (Peduzzi et al., 1996).

\section{Análisis estadístico}

Los resultados de variables cualitativas se expresaron como frecuencias y porcentajes. Las variables cuantitativas, mediante media y desviación standard. Se utilizó medidas de tendencia y distribución para describir los grupos. Se utilizó el t-test y ANOVA para analizar las variables continuas, pruebas de Chi cuadrado para comparar las variables cualitativas. Regresión logística binaria para el análisis multivariado, entre sexo, edad, hora de acostarse, hora de levantarse, latencia de Inicio de sueño, horas de sueño nocturno $<6$ horas y $>$ 10 horas, diagnóstico de depresión, ansiedad y malos dormidores. Se consideró significativa una $\mathrm{p}<0.05$. Se usó SPSS 18 (Chicago SPSS Inc.).

\section{Resultados}

Se completaron 2798 encuestas. Se incluyeron 2594 personas, excluyéndose 204 por formulario incompleto o por vivir en el extranjero al momento de completar la encuesta. El 31\% fueron hombres $(n=803)$. Edad: $18-85$, media $42 \pm 13$. Edad $\geq 65$ años 5,4\% $(n=141)$. El 66.6\% residía en la provincia de Buenos Aires (en el cono urbano: $28,7 \%$ y en el interior de la provincia: $37,9 \%)$.
La mayor participación del interior correspondió a las provincias de Santa Fe con 6,3\%, Catamarca: 3\%, Córdoba: $2,9 \%$, Chubut: $2,7 \%$. Jujuy fue la única provincia de la cual no se obtuvo datos. El $67.5 \%$ $(n=1751)$ estaba empleado y de ellos el 31.7\% ( $n=823)$ trabajaban en salud de los cuales $n=515$ o sea el $19,9 \%$ eran mujeres. Al dividir por géneros las mujeres que no trabajaban fueron $23,9(n=619)$, y trabajan pero no en salud el 25,3\% ( $n=657)$. Las características generales de la población evaluada y de los cuestionarios/ preguntas analizadas, se observan en la tabla 1.

En la tabla 2 se analizó las diversas variables de sueño por PSQI, hábitos de sueño, la presencia de síntomas de depresión por PHQ-9 y TAG por GAD-7.

En la tabla 3 analizamos PSQI según género mediante ANOVA donde se observó que las mujeres son más malas dormidoras, tardan más en dormirse, se levantan más tarde y duermen más horas, les cuesta dormir la primer media hora, tienen más despertares, roncan más, tienen más pesadillas, y otras razones que fragmentan su sueño. Refirieron mala a muy mala calidad de sueño, toman más medicamentos para dormir, y tienen peor estado de ánimo para hacer las cosas al despertarse, que los hombres.

Además, comparamos la calidad de sueño entre adultos y personas de la tercera edad donde observamos llamativamente que no hubo diferencias en ser malos dormidores, pero los de mayor edad refirieron acostarse y dormirse antes, les cuesta dormir la primera media hora, roncan menos, refirieron menos pesadillas, no tan mala calidad del sueño, toman menos medicación para dormir, y no tienen tantos problemas con el ánimo para hacer las cosas.

En la tabla 4, se comparó mediante ANOVA los cuestionarios de PSQI, PHQ-9 y GAD observándose que a mayor depresión y ansiedad, la calidad del sueño es significativamente peor.

En la tabla 5 se realizó análisis de regresión logística multivariado comparando depresión y ansiedad con múltiples variables relacionadas al sueño y se observó que tener menos de 55 años, dormir más de 10 horas, ser mal dormidor y presentar ansiedad se asoció a síntomas de depresión. Además ser mal dormidor y presentar síntomas de depresión se asoció a síntomas de ansiedad.

Al comparar mediante ANOVA los trabajadores, trabajadores de salud con los no trabajadores (ver tabla 6) encontramos diferencia significativa en los No trabajadores dado que se acostaban más tarde, se levantaban más tarde, dormían más horas, la latencia de inicio de sueño era mayor, y también mayor la percepción de somnolencia diurna. El puntaje de PQH9 y ser 
Tabla I. Características generales de la población y análisis de variables relacionadas con el sueño, depresión, ansiedad

\begin{tabular}{|c|c|c|c|c|c|}
\hline Variables analizadas & $\mathrm{n}$ & Mínimo & Máximo & Media & $\mathrm{DE}$ \\
\hline Edad & 2594 & 18 & 85 & 42 & 13 \\
\hline Horas de Exposición a COVID-19 & 761 & 0 & 72 & 7 & 12,40 \\
\hline PSQI \# IHora de Acostarse (h/min) & 2594 & 20:00 & 04:00 & 00:02 & $\mathrm{I}: 22$ \\
\hline PSQI \# 2 Latencia de Inicio de Sueño (min) & 2594 & 0 & 240 & 37,82 & 40,35 \\
\hline PSQI \# 3 Hora de Levantarse (h/min) & 2594 & 04:00 & 14:00 & 08:29 & $1: 38$ \\
\hline PSQI \# 4 Horas del Sueño (h/min) & 2594 & 4 & 12 & 7:00 & $1: 18$ \\
\hline PSQI \# I y 3.Tiempo en Cama (h/min) & 2594 & 04:00 & $14: 00$ & $8: 26$ & $\mathrm{I}: 25$ \\
\hline PSQI: Eficiencia del Sueño (\%) & 2594 & 40 & 100 & 83,49 & 13,18 \\
\hline Escala analógica Visual de Calidad de Sueño & 2594 & 1 & 10 & 6,56 & 2,10 \\
\hline Escala analógica Visual de Somnolencia Diurna & 2594 & I & 10 & 3,92 & 2,50 \\
\hline TTSS (h/min)* & 2594 & 4 & 12 & 7:00 & $1: 18$ \\
\hline Tiempo que demora en Levantarse (min) & 2594 & 0 & 240 & 23,06 & 24,01 \\
\hline Siestas: duración en minutos & $|47|$ & 5 & 180 & 67,99 & 36,3 \\
\hline PSQI puntaje & 2594 & 0 & 19 & 6,35 & 3,45 \\
\hline PHQ-9 - Puntaje & 2594 & 0 & 27 & 6,39 & 4,62 \\
\hline GAD - Puntaje & 2594 & 0 & 21 & 5,58 & 4,14 \\
\hline
\end{tabular}

*TTSS:Tiempo Total de Sueño Subjetivo

malos dormidores por PSQI total, también peor, estadísticamente significativo comparado con los trabajadores. Los trabajadores de salud puntuaron significativamente, más en el GAD-7, que los no trabajadores.

\section{Discusión}

Realizamos por primera vez una encuesta sobre calidad de sueño, ansiedad y síntomas depresivos durante la pandemia por COVID-19 en Argentina. No encontramos que dichos aspectos hayan sido estudiados en otros países pero encontramos un trabajo de Huang en China, que fue publicado un día antes que la distribución de nuestra encuesta (Huang, y Zhao, 2020), con resultados en su mayoría disímiles a los nuestros. Nuestra población estuvo integrada por dos tercios de residentes de la provincia de Buenos Aires y Ciudad de Buenos Aires. Casi 2 tercios de la población tenía empleo, de los cuales más de un tercio trabajaba en el ámbito de la salud y 5,4\% pertenecía a la tercera edad.

$\mathrm{Al}$ analizar la calidad del sueño encontramos que más de la mitad de nuestra población refirieron ser malos dormidores a diferencia de la población asiática donde el porcentaje fue muy inferior al nuestro (Huang, y Zhao, 2020). La mala calidad de sueño se asoció con varios factores como: aumento de latencia al inicio del sueño y de los despertares nocturnos, aunque el tiempo total de sueño subjetivo estuvo dentro de los valo- res considerados como normales para adultos (Vyas, y Suno, 2020). Sólo los trabajadores de salud manifestaron como otra razón, dormir menos horas. Se sabe que dormir menos tiempo de lo adecuado, predispone a estrés crónico y mayor angustia (Lee et al., 2018).

$\mathrm{Al}$ analizar la calidad del sueño en la tercera edad, detectamos que si bien los sujetos refirieron mala calidad de sueño, se acostaban más temprano y dormían antes, constatándose adelanto de fase probablemente por la edad (Paul et al., 2011), pero no se pueden descartar otros factores visibles en la cuarentena como falta de exposición a la luz solar, sedentarismo y/o falta de relaciones sociales. No encontramos reportes en la literatura que hayan analizado el sueño en esta población durante la pandemia.

El sub-análisis de la calidad del sueño en mujeres demostró en forma significativa que tenían una mala calidad de sueño, a pesar de que dormían más horas pero su sueño estaba más fragmentado por prácticamente todos los motivos mencionados, e incluso refirieron roncar con mayor frecuencia que los varones. Además, refirieron consumir significativamente más medicación para poder dormir. Al realizar el análisis multivariado encontramos también que las mujeres que tenían mala calidad de sueño, dormían más de 10 horas, experimentaban ansiedad, asociada fuertemente a presentar síntomas de depresión, sobre todo 
Tabla 2. Análisis de variables de sueño y algunas variables de PSQI, hábitos de sueño, depresión (PHQ-9) y ansiedad (GAD)

\begin{tabular}{|c|c|c|c|}
\hline Valoración de sueño & Características evaluadas & $\mathrm{n}$ & $\%$ \\
\hline \multirow{2}{*}{ Calidad de sueño por PSQI } & PSQI total $\leq 5$ & 1219 & 47 \\
\hline & PSQI total >5 & 1375 & 53 \\
\hline \multirow{4}{*}{ Clasificación obtenida de PSQI } & Normal & 892 & 34,4 \\
\hline & Requiere Tratamiento médico & 807 & 31,1 \\
\hline & Requiere Atención y tratamiento & 841 & 32,4 \\
\hline & Problema grave de sueño & 54 & 2,1 \\
\hline PSQI \# 5a. No dormir primer media hora & $\geq \mathrm{I} / 7$ días & 1220 & 1220 \\
\hline PSQI \# 5b. Despertarse durante noche & $\geq 1 / 7$ días & 1514 & 58,4 \\
\hline PSQI \# 5c. Necesidad de ir al Baño & $\geq 1 / 7$ días & 1336 & 51,5 \\
\hline PSQI \# 5d. No poder Respirar bien & $\geq$ I/7 días & 219 & 8,4 \\
\hline PSQI \# 5e.Tos/Roncar ruidosamente & $\geq 1 / 7$ días & 400 & 15,4 \\
\hline PSQI \# 5f. Sentir Frío & $\geq 1 / 7$ días & 630 & 24,3 \\
\hline PSQI \# 5g. Sentir Calor & $\geq$ I/7 días & 665 & 25,6 \\
\hline PSQI \# 5h.Tener Pesadillas & $\geq 1 / 7$ días & 593 & 22,9 \\
\hline PSQI \# 5i. Tener Dolores & $\geq 1 / 7$ días & 695 & 695 \\
\hline PSQI \# 5j. Otras razones & $\geq 1 / 7$ días & 466 & 18,0 \\
\hline \multirow{2}{*}{ PSQI \# 6. Calificación de Calidad de sueño } & Muy Buena y bastante buena & 1703 & 65,6 \\
\hline & Bastante Mala y muy mala & 891 & 34,3 \\
\hline PSQI \# 7.Tomar medicación recetada/no recetada para dormir & $\geq 1 / 7$ días & 357 & 13,8 \\
\hline PSQI \# 8. Problemas para permanecer despierto & $\geq 1 / 7$ días & 222 & 8,6 \\
\hline \multirow{4}{*}{ PSQI \# 9. Tener ánimo para realizar actividades al levantarse } & Ningún Problema & 1203 & 46,4 \\
\hline & Poco Problema & 881 & 881 \\
\hline & Moderado Problema & 422 & 16,3 \\
\hline & Mucho Problema & 88 & 3,4 \\
\hline \multirow{4}{*}{ Hábito de Siesta } & No duerme siesta & 1114 & 42,9 \\
\hline & Si duerme siesta & 1480 & 57,1 \\
\hline & $<3 / 7$ & 836 & 32,2 \\
\hline & $>3 / 73$ & 644 & 24,8 \\
\hline \multirow{3}{*}{ Frecuencia de Siesta } & Menor frecuencia & 630 & 24,3 \\
\hline & Igual frecuencia & 516 & 19,9 \\
\hline & Mayor frecuencia & 334 & 12,9 \\
\hline \multirow{3}{*}{ Duración de Siesta } & Menor duración & 360 & 13,9 \\
\hline & Igual duración & 753 & 29,0 \\
\hline & Mayor duración & 367 & 14,1 \\
\hline \multirow{3}{*}{ Horario de Acostarse Noche } & Más Temprano & 826 & 31,8 \\
\hline & Igual & 1083 & 41,8 \\
\hline & Más Tarde & 685 & 26,4 \\
\hline \multirow{2}{*}{ PHQ-9 Total } & Puntaje 0-9 (no o dudoso) & 2046 & 78,9 \\
\hline & Puntaje 10-27(diagnóstico) & 548 & 21,1 \\
\hline \multirow{5}{*}{ Puntajes de PHQ-9 } & Puntaje de 0-4 & 1007 & 38,8 \\
\hline & Puntaje de 5-9 & 1039 & 40 \\
\hline & Puntaje de $10-14$ & 384 & 14,8 \\
\hline & Puntaje de 15-19 & 129 & 5,0 \\
\hline & Puntaje de 20-27 & 35 & 1,3 \\
\hline GAD-7 Total & Positivo (6 a $2 \mathrm{I}$ ) & 1135 & 43,8 \\
\hline \multirow{4}{*}{ Grado de ansiedad } & Ausente $(0 \mathrm{a} 4)$ & 1161 & 44,8 \\
\hline & Leve $(5$ a 10$)$ & 1114 & 42,9 \\
\hline & Moderada (II a I5) & 234 & 9,0 \\
\hline & Severa ( 16 a 21$)$ & 85 & 3,3 \\
\hline
\end{tabular}


Tabla 3. Análisis comparativo de PSQI según género y edad mediante Prueba de T

\begin{tabular}{|c|c|c|c|c|c|c|}
\hline & \multicolumn{2}{|c|}{ Género } & \multirow{3}{*}{$\mathrm{P}$} & \multicolumn{2}{|c|}{ Edad } & \multirow{3}{*}{$\mathrm{P}$} \\
\hline & $\begin{array}{c}\text { Femenino } \\
n=|79|\end{array}$ & $\begin{array}{c}\text { Masculino } \\
n=803\end{array}$ & & $\begin{array}{c}<65 \text { Años } \\
\mathrm{n}=2453\end{array}$ & $\begin{array}{c}\geq 65 \text { Años } \\
n=|4|\end{array}$ & \\
\hline & Media \pm DE & Media \pm DE & & Media \pm DE & Media $\pm \mathrm{DE}$ & \\
\hline PSQI Puntaje Total & $6,64 \pm 3,51$ & $5,71 \pm 3,23$ & 0,00 & $6,36 \pm 3,43$ & $6,26 \pm 3,93$ & 0,09 \\
\hline PSQI I: Hora Acostarse (hh:mm) & $23: 58 \pm 1: 21$ & $00: 13 \pm 1: 23$ & 0,00 & $00: 04 \pm 1: 23$ & $23: 47 \pm 1: 11$ & 0,02 \\
\hline PSQI 2: Latencia Inicio (min) & $40,09 \pm 41,9$ & $32,49 \pm 35,5$ & 0,00 & $38,3 \pm 40,04$ & $28,54 \pm 41,38$ & 0,00 \\
\hline PSQI 3: Hora Levantarse (hh:mm) & $08: 32 \pm 1: 37$ & $08: 23 \pm 1: 40$ & 0,03 & $08: 29 \pm 1: 39$ & $08: 26 \pm 1: 21$ & 0,70 \\
\hline \multirow[t]{3}{*}{ PSQI 4: Duración Sueño (hh:mm) } & $07: 03 \pm 1: 20$ & $06: 55 \pm 1: 13$ & 0,00 & $07: 06 \pm 1: 19$ & $06: 57 \pm 1: 16$ & 0,57 \\
\hline & \multicolumn{2}{|c|}{ Género } & & \multicolumn{2}{|c|}{ Edad } & \\
\hline & $\begin{array}{c}\text { Femenino } \\
n=|79|\end{array}$ & $\begin{array}{l}\text { Masculino } \\
\mathrm{n}=803\end{array}$ & $\mathrm{P}$ & $\begin{array}{l}<65 \text { Años } \\
\mathrm{n}=2453\end{array}$ & $\begin{array}{c}\geq 65 \text { Años } \\
n=|4|\end{array}$ & $P$ \\
\hline PSQI 5a. No dormir primer media hora $\geq \mathrm{I} / 7$ & $33,7 \%$ & $13,3 \%$ & 0,00 & $45 \%$ & $2 \%$ & 0,01 \\
\hline PSQI 5b. Despertarse durante noche $\geq \mathrm{I} / 7$ & $41,6 \%$ & $16,8 \%$ & 0,00 & $55 \%$ & $3,4 \%$ & 0,31 \\
\hline PSQI 5c. Necesidad de ir al Baño $\geq$ I/7 & $36 \%$ & $15,5 \%$ & 0,28 & $47,3 \%$ & $4,2 \%$ & 0,00 \\
\hline PSQI 5d. No poder Respirar bien $\geq 1 / 7$ & $6,1 \%$ & $2,4 \%$ & 0,30 & $8,1 \%$ & $0,4 \%$ & 0,55 \\
\hline PSQI 5e.Tos o Roncar $\geq 1 / 7$ & $9,3 \%$ & $6,2 \%$ & 0,00 & $14 \%$ & $1,5 \%$ & 0,00 \\
\hline PSQI 5f. Sentir Frío $\geq$ I/7 & $18,5 \%$ & $5,8 \%$ & 0,00 & $23,2 \%$ & $1 \%$ & 0,14 \\
\hline PSQI 5g. Sentir Calor $\geq \mathrm{I} / 7$ & $19,1 \%$ & $6,6 \%$ & 0,00 & $24,4 \%$ & $1,2 \%$ & 0,30 \\
\hline PSQI 5h. Tener Pesadillas $\geq$ I/7 & $17,4 \%$ & $5,5 \%$ & 0,00 & $21,9 \%$ & $0,9 \%$ & 0,09 \\
\hline PSQI 5i.Tener Dolores $\geq$ I/7 & $21,1 \%$ & $5,7 \%$ & 0,00 & $25,2 \%$ & $1,6 \%$ & 0,40 \\
\hline PSQI 5j. Otras Razones $\geq$ I/7 & $14,4 \%$ & $3,6 \%$ & 0,00 & $17 \%$ & $1 \%$ & 0,94 \\
\hline PSQI 6. Calidad Sueño Mala o Bastante Mala & $25,1 \%$ & $9,3 \%$ & 0,00 & $33,1 \%$ & $1,2 \%$ & 0,00 \\
\hline PSQI 7.Tomar Hipnóticos $\geq \mathrm{I} / 7$ & $10,1 \%$ & $3,6 \%$ & 0,04 & $12,2 \%$ & $1,5 \%$ & 0,00 \\
\hline PSQI 8. Problemas estar despierto $\geq \mathrm{I} / 7$ & $6,2 \%$ & $2,4 \%$ & 0,31 & $8,4 \%$ & $0,2 \%$ & 0,03 \\
\hline $\begin{array}{l}\text { PSQI 9. Mucho o Medio Problema de } \\
\text { Ánimo para realizar actividades }\end{array}$ & $14,5 \%$ & $5,2 \%$ & 0,02 & $19,2 \%$ & $0,5 \%$ & 0,00 \\
\hline PHQ-9 > 9 (Depresión) & $17 \%$ & $4,2 \%$ & 0,00 & $29,7 \%$ & $0,5 \%$ & 0,00 \\
\hline GAD > 5 (Ansiedad) & $33,4 \%$ & $19,3 \%$ & 0,00 & $42,3 \%$ & $1,5 \%$ & 0,00 \\
\hline
\end{tabular}

en las menores de 65 años. Tanto la genética, como la fisiología de cada individuo, los factores psicológicos e incluso los cambios en el ritmo sueño y vigilia (Soria, y Urretavizcaya, 2009), son motivos que predisponen a desarrollar depresión; como así también la disminución de amplitud de la temperatura estacional y la alteración de la secreción de algunas hormonas (Linkowski et al., 1994, Souetre et al., 1988) actuando como causas asociadas que justificarían el mal dormir y la depresión. Creemos que los cambios producidos por el confinamiento como la variación en los horarios, los disturbios en la rutina social/conductual y la falta de exposición lumínica, puede justificar esa asociación encontrada en la Argentina.

También encontramos que los síntomas depresivos se asociaron ampliamente a trastornos de ansiedad, a mala calidad del sueño, sobre todo en no trabajadores y en los que dormían más de 10 horas (hecho observado en la práctica clínica pero que aún es motivo de estudio).

Más de $1 / 3$ de los participantes mostraron trastornos de ansiedad, en trabajadores sobre todo de salud y en mujeres (Gao et al., 2020). Coincidimos con otros autores (Furer et al., 1997) que la ansiedad podría estar relacionada con preocupación por la infección y temor a que la pandemia fuera difícil de controlar. Pero también pensamos que los mayores de 65 años pudiesen estar preocupados por miedo al contagio; sin embargo, no encontramos valores significativos al respecto, quizá por el hecho de que el confinamiento no modifica y/o mantiene estable sus actividades, su rutina y su economía. Además, uno de los sesgos de nuestro estudio es haber contado con una población pequeña, y no haber 
Tabla 4. Relación entre los cuestionarios de Depresión (PHQ-9), Ansiedad (GAD) y Calidad de sueño (PSQI) mediante ANOVA Univariado

\begin{tabular}{lcccc}
\hline Cuestionarios analizados & $\mathrm{n}$ & Media PSQI & DE & $\mathrm{P}$ \\
\hline PHQ-9: 0-4 puntos & 1007 & 4,47 & 2,61 & \\
\hline PHQ-9: 5-9 puntos & 1039 & 6,80 & 3,04 & \\
\hline PHQ-9: $10-14$ puntos & 384 & 8,41 & 3,39 & 0,000 \\
\hline PHQ-9: I5-19 puntos & 129 & 9,79 & 3,70 & \\
\hline PHQ-9: 20-27 puntos & 35 & 11,83 & 3,17 & \\
\hline GAD 0-4: Normal & 1161 & 4,84 & $2,8 \mid$ & \\
\hline GAD 5- 10: Leve & 1114 & 7,18 & 3,33 & \\
\hline GAD II- I5: Moderada & 234 & 8,79 & 3,33 & 0,000 \\
\hline GAD I6-2I: Severa & 85 & 9,42 & 3,88 & \\
\hline
\end{tabular}

Tabla 5. Análisis multivariado de regresión logística binaria de calidad de sueño por PSQI, depresión por PHQ-9 y ansiedad por GAD

\begin{tabular}{|c|c|c|c|c|c|c|c|c|c|}
\hline \multirow{2}{*}{ Variables analizadas } & \multicolumn{3}{|c|}{ Depresión por PHQ-9 } & \multicolumn{3}{|c|}{ Ansiedad por GAD } & \multicolumn{3}{|c|}{ Calidad de sueño por PSQI } \\
\hline & OR & IC95 & $P$ & OR & IC95 & $P$ & OR & IC95 & $P$ \\
\hline Sexo Femenino & $\mathrm{I}, 54$ & $1,19-1,99$ & 0,001 & $\mathrm{I}, 50$ & $1,23-1,82$ & 0,000 & 1,29 & $\mathrm{I}, 06-\mathrm{I}, 57$ & 0,010 \\
\hline Edad $<55$ años & 2,19 & $1,52-3,14$ & 0,000 & $\mathrm{I}, 73$ & $|, 35-2,2|$ & 0,000 & 0,79 & $0,62-1,00$ & 0,053 \\
\hline Hora Acostarse $>24: 00$ & 1,26 & $0,99-I, 56$ & 0,057 & 1,16 & $0,95-1,40$ & 0,145 & 0,78 & $0,64-0,95$ & 0,011 \\
\hline Hora Levantarse $>08: 00$ & $\mathrm{I}, 07$ & $0,82-1,39$ & 0,623 & 0,76 & $0,62-0,95$ & 0,013 & $|, 5|$ & $1,22-1,88$ & 0,000 \\
\hline Latencia Inicio > $30 \mathrm{~min}$ & 1,28 & $|, 28-|, 0 \mid$ & 0,039 & $\mathrm{I}, 0 \mathrm{I}$ & $0,84-1,23$ & 0,901 & 3,55 & $2,96-4,26$ & 0,000 \\
\hline Dormidor Corto (< 6 hs) & $\mathrm{I}, 80$ & $1,32-2,46$ & 0,000 & $|, 2|$ & $0,90-1,62$ & 0,201 & 3,61 & $2,59-5,04$ & 0,000 \\
\hline Dormir Largo (> 10 hs) & 2,23 & $|, 2|-4,09$ & 0,010 & 0,61 & $0,35-1,07$ & 0,082 & 0,75 & $0,44-1,26$ & 0,275 \\
\hline Hábito de Siestas & 1,33 & $1,06-1,66$ & 0,013 & 0,89 & $0,74-1,07$ & 0,225 & $\mathrm{I}, 2 \mathrm{I}$ & $I, 0 I-I, 46$ & 0,038 \\
\hline Ronquidos ( $\geq \mathrm{I} / 7$ ) & $\mathrm{I}, 30$ & $0,96-0,71$ & 0,802 & $\mathrm{I}, \mathrm{II}$ & $0,86-1,44$ & $0,4 \mid 2$ & 0,58 & $0,44-0,75$ & 0,000 \\
\hline Pesadillas $(\geq 1 / 7)$ & $\mathrm{I}, 56$ & $1,23-1,97$ & 0,000 & $\mathrm{I}, 8 \mathrm{I}$ & $1,45-2,25$ & 0,000 & $\mathrm{I}, 72$ & $1,37-2,16$ & 0,000 \\
\hline PSQI > 5 & 2,30 & I,77-2,99 & 0,000 & 2,83 & $2,32-3,44$ & 0,000 & & & \\
\hline PHQ-9 >9 & & & & 6,77 & $5,24-8,75$ & 0,000 & 2,31 & I,78-3,0। & 0,000 \\
\hline GAD > 5 & 6,77 & $5,24-8,76$ & 0,000 & & & & 2,82 & $2,32-3,43$ & 0,000 \\
\hline Coeficiente Cox y Snell & & $21,5 \%$ & & & $23,7 \%$ & & & $24,8 \%$ & \\
\hline Coeficiente Nagelkerke & & $33,4 \%$ & & & $31,8 \%$ & & & $33,1 \%$ & \\
\hline
\end{tabular}

diferenciado si vivían en un geriátrico, aparte de que el estudio se hizo al mes de declarada la cuarentena reportándose entonces leves incrementos.

El origen multicausal de los trastornos de ansiedad, la activación amigdalina/hipocampal sobre sistemas orexinérgicos/monoaminérgicos que produciría la activación del mecanismos provocadores de microdespertares, constituyendo, probablemente, el mecanismo fisiopatológico relacionado al estado de "alerta" y a la ausencia de síntomas de somnolencia, que pudimos constarlo en nuestra población (Breslau et al., 1996; Ford, y Kamerow, 1989; Franzen, y Buysse, 2008; RTM IV, 2012).
En la etiopatogenia también se plantean factores genéticos, compartidos con la depresión, lo que explicaría la elevada comorbilidad de ambos trastornos (Cañellas, y Collado, 2012), como encontramos en nuestro estudio, en el cual, además, se triplicó su asociación en los malos dormidores. Por ello, creemos que reforzar la acción de los zeitgebers externos, como incentivar la actividad física (Mead et al., 2009), restaurar lentamente los ritmos sociales, promover la exposición a la luz, mantener horarios regulares de sueño y de alimentación (Most et al., 2010; Terman, y Terman, 2004), colaborarían en mejorar los cambios de humor 
Tabla 6. Análisis variables en no trabajadores, trabajadores y trabajadores de salud, mediante test de ANOVA univariado

\begin{tabular}{|c|c|c|c|c|c|c|c|}
\hline \multirow{2}{*}{ Variables analizadas } & \multicolumn{2}{|c|}{ NO Trabajador $(n=844)$} & \multicolumn{2}{|c|}{ Trabajador $(n=\mid 750)$} & \multicolumn{2}{|c|}{ Trabajador Salud $(n=7 \mid 0)$} & \multirow{2}{*}{$P$} \\
\hline & Media & $\mathrm{DE}$ & Media & $\mathrm{DE}$ & Media & $\mathrm{DE}$ & \\
\hline Edad (años) & 41,18 & $\pm 16,16$ & 41,13 & $\pm 11,99$ & 42,95 & $\pm 10,48$ & $0,008^{a}$ \\
\hline Hora de Acostarse (hh:mm) & $00: 21$ & $\pm 1: 32$ & $00: 01$ & $\pm 1: 19$ & $23: 42$ & $\pm 1: 09$ & $0,000^{b}$ \\
\hline Hora de Levantarse (hh:mm) & $09: 13$ & $\pm 1: 39$ & $08: 26$ & $\pm 1: 28$ & $07: 41$ & $\pm 1: 25$ & 0,000 \\
\hline Duración Sueño (hh:mm) & $7: 16$ & $\pm 1: 22$ & $7: 01$ & $\pm 1: 04$ & $6: 42$ & $\pm 1: 13$ & $0,000^{\circ}$ \\
\hline Tiempo en Cama (hh:mm) & $8: 52$ & $\pm 1: 23$ & $8: 25$ & $\pm 1: 23$ & $7: 59$ & $\pm 1: 22$ & $0,000^{\circ}$ \\
\hline Latencia Inicio Sueño (min) & 43,77 & $\pm 47,24$ & 36,11 & $\pm 37,35$ & 32,96 & $\pm 33,67$ & 0,000 \\
\hline Latencia de Levantarse (min) & 25,69 & $\pm 25,76$ & 23,18 & $\pm 24,52$ & $|9,7|$ & $\pm 18,76$ & 0,000 \\
\hline $\begin{array}{l}\text { Escala analógica visual de Calidad de } \\
\text { Sueño (I al I0) }\end{array}$ & 6,51 & $\pm 2,17$ & 6,62 & $\pm 2,12$ & 6,53 & $\pm 2,0 \mathrm{I}$ & 0,487 \\
\hline $\begin{array}{l}\text { Escala analógica visual de Somnolencia } \\
\text { Diurna (I al I0) }\end{array}$ & 4,04 & $\pm 2,55$ & 4,03 & $\pm 2,52$ & 3,64 & $\pm 2,41$ & $0,002^{h}$ \\
\hline PSQI - Calidad de Sueño & 6,60 & $\pm 3,53$ & 6,19 & $\pm 3,41$ & 6,29 & $\pm 3,42$ & $0,032^{k}$ \\
\hline PHQ-9 - Depresión & 6,73 & $\pm 4,99$ & 6,06 & $\pm 4,39$ & 6,47 & $\pm 4,45$ & 0,007 \\
\hline GAD-7 - Ansiedad & 5,51 & $\pm 4,13$ & 5,37 & $\pm 4,09$ & 5,99 & $\pm 3,98$ & 0,007 \\
\hline
\end{tabular}

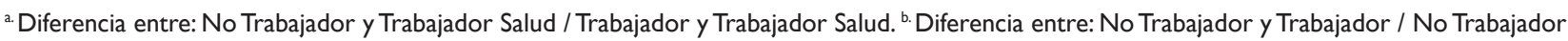
y Trabajador Salud / Trabajador y Trabajador Salud. c. Diferencia entre: No Trabajador y Trabajador / No Trabajador y Trabajador Salud / Trabajador y Trabajador Salud. ${ }^{d}$ Diferencia entre: No Trabajador y Trabajador / No Trabajador y Trabajador Salud / Trabajador y Trabajador Salud. e. Diferencia entre: No Trabajador y Trabajador / No Trabajador y Trabajador Salud / Trabajador y Trabajador Salud. f. Diferencia entre: No Trabajador y Trabajador / No

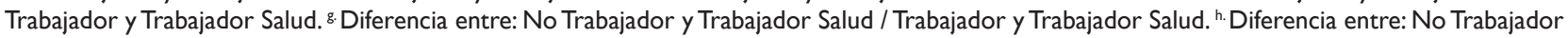
y Trabajador Salud / Trabajador y Trabajador Salud. i. Diferencia entre: No Trabajador y Trabajador. i. Diferencia entre:Trabajador y Trabajador Salud. ${ }^{k}$. Diferencia entre: No Trabajador y Trabajador

en la población durante la pandemia. Se debe considerar que, si bien fue detectado un pequeño porcentaje de casos severos de ansiedad (3\%), pueden aparecer síntomas de estrés postraumático, que estaría correlacionado con la falta de sueño (Kobayashi et al., 2007), pero la prevalencia de trastorno de ansiedad fue muy superior a la población asiática, por lo que nos obliga a utilizar herramientas para mejorar el sueño y tratarlos precozmente a nivel nacional.

Los trabajadores de la salud merecieron un análisis aparte dado que constatamos que además de ser malos dormidores, asociaron elevados síntomas de ansiedad. Probablemente, el miedo a contagiarse, el estrés crónico y la angustia psicológica (Lu et al., 2006) los predispusieron a esos fenómenos, en una población mayoritariamente femenina.

Los sesgos de este estudio fueron varios debido que utilizamos un método de encuesta basado en la web por lo que el muestreo de nuestro estudio fue voluntario pero a actuó a favor, la cantidad de personas que participaron que superó ampliamente el cálculo de la muestra poblacional requerida. Otro sesgo, de los varios que posee el trabajo, es no haber interrogado sobre el consumo de alcohol como inductor de sueño, la cantidad de horas de exposición a la luz, y el haber podido evaluar mayor cantidad de población de la tercera edad, aunque, también debe considerarse que es el primer trabajo que diferencia a esta población durante la pandemia de COVID-19, incluyendo personas que no se han enfermado, y mostrando lo habitual que es disfunción del ritmo circadiano por adelanto de fase (se acuestan antes, se duermen antes, duermen aproximadamente 6 a 7 horas).

\section{Conclusiones}

Identificamos cambios en el sueño y la salud mental durante la fase inicial del confinamiento por COVID-10 en la Argentina, en población de adultos. La calidad del sueño estuvo afectada en más de la mitad de la población, constatándose importante prevalencia de síntomas de ansiedad y en menor medida síntomas de depresión. Los trabajadores de salud presentaron más síntomas de ansiedad que el resto. La tercera edad mostró cambios en el sueño.

Nuestros hallazgos se pueden utilizar para formular intervenciones en el sueño para mejorar la salud mental y la capacidad de recuperación psicológica post pandemia. 


\section{Agradecimientos}

A los médicos designados como delegados regionales o aquellos médicos que colaboraron activamente en distribuir la encuesta, por orden alfabético: Borghini, M.; Camji, J.; Castellino, G.; Coronel, M.; Fernández, M.C.; Ferro, R.; Franceschini, C.; Galíndez, F.; Heitz, I.; Larrateguy, L.; Luján, S.; Lucero, C.; Ruffa, E.; Ruggiero, M.; Sartori, V., Smurra, M.; Questa, M.; Matejik, P. y a todos los asociados que colaboraron de una u otra manera.

\section{Conflictos de intereses: los autores declaran no tener conflictos de intereses.}

\section{Referencias bibliográficas}

Breslau, N., Roth, T., Rosenthal, L., Andreski, P. (1996). Sleep disturbance and psychiatric disorders: a longitudinal epidemiological study of young adults. Biol Psychiatry, 39, 411-418.

Buysse, D., Reynolds, C., Monk, T. et al. (1989). The Pittsburgh Sleep Quality Index: a new Instrument for Psychiatric Practice and Reseach. Psychiatric Res, 28, 193-213.

Cañellas, F., Collado, C. (2012). Enfermedades psiquiátricas y sueño. En Sociedad Española de Sueño, Tratado de Medicina del sueño. Editorial Panamericana, pp. 701-725.

Dong, L., Boueym J. (2020). Public mental health crisis during COVID-19 pandemic, China. Emerg Infect Dis, 26(7), 1616-1618. https://doi. org/10.3201/eid2607.200407

Escobar-Córdoba, F., Eslava-Schmalbach, J. (2005). Validación colombiana del índice de calidad de sueño de Pittsburgh España. Revista de Neurología, 40, 150-5.

Ford, D. E., \& Kamerow, D. B. (1989). Epidemiologic study of sleep disturbances and psychiatric disorders. An opportunity for prevention? JAMA, 262(11), 1479-1484. https://doi.org/10.1001/jama.262.11.1479

Franzen, P.L., Buysse, D.J. (2008). Sleep disturbances and depression: risk relationships for subsequent depression and therapeutic implications. Clin Neurosci, 10(4), 473-481.

Furer, P., Walker, J.R., Chartier, M.J., Stein, M.B. (1997). Hypochondriacal concerns and somatization in panic disorder. Depress Anxiety, 6, 78-85. https://doi: 10.1002/(sici)1520-6394(1997)6:23.0.co;2-1.

Gao, W., Ping, S., Liu, X. (2020). Gender differences in depression, anxiety, and stress among college students: a longitudinal study from China. $J$ Affect Disord, 263, 292-300. https://doi.org/10.1016/j.jad.2019.11.121.

García-Campayo, J., Zamorano, E., Ruiz, M.A., Pardo, A., Pérez-Páramo, S., López-Gómez, V., Freire, O., Rejas, F. (2010). Cultural adaptation into Spanish of the generalized anxiety disorder-7/GAD-7 scales a screening tool. Health and quality of Life Outcomes, 20(8):8. https://doi: 10.1186/1477-7525-8-8

Ho, C. S., Chee, C. Y., Ho, R.C. (2020). Mental health strategies to combat the psychological impact of COVID-19 beyond paranoia and panic. Ann Acad Med Singap, 49(1), 1.

Huang, Y., Zhao, N. (2020). Generalized anxiety disorder, depressive symptoms and sleep quality during COVID-19 outbreak in China: a web-based cross-sectional survey. Psychiatry Research, 288, 11-29 https:// doi: 10.1016/j.psychres.2020.112954

Jimenez-Genchi, A., Monteverde-Maldonado, E. (2008). Confiabilidad y análisis factorial de la versión en español del índice de calidad del sueño de Pittsburg en pacientes psiquiátricos. Gac Med Mex, 144 (6): 491-6.

Kevin, J., Gaston, K. J., Davies, T.W., Nedelec, S. L., Holt, L. A. (2017). Impacts of Artificial Light at Night on Biological Trimings. Annual Review of Ecology, Evolution, and Systematics, 48, 49-68. https://doi.org/10.1146/ annurev-ecolsys-110316-022745
Kobayashi, I., Boarts, J.M., Delahanty, D.L. (2007). Polysomnographically measured sleep abnormalities in PTSD: a meta-analytic review. Psychophysiology, 44, 660-669. https://doi.org/10.1111/j.1469- 8986.2007.537.x

Kroenke, K., Spitzer, R. L. (2002). The PHQ-9: a new depression diagnostic and severity measure. Psychiatric Annals, 32509-521. http://www.lphi.org/ LPHIadmin/uploads/.PHQ-9-Review-Kroenke-63754.

Kroenke, K., Spitzer, R. L., Williams, J. B. W. (2001). The PHQ-9: validity of a brief depression severity measure. J Gen Intern Med, 16(9), 606-613.

Lee, SM., Kang, WS., Cho, A.R., Kim, T., Park, J.K. (2018). Psychological impact of the 2015 MERS outbreak on hospital workers and quarantined hemodialysis patients. Compr Psychiatry, 87, 123-127. https://doi:org/10.1016/j.comppsych.2018.10.003.

Linkowski, P., Kerkhofs, M., Van Onderbergenm A. et al. (1994). The 24hour profiles of cortisol, prolactin, and growth hormone secretion in mania. Arch Gen Psyiatry, 51, 616-24.

Lu, Y.C., Shu, B.C., Chang, Y.Y., Lung, F.W. (2006). The mental health of hospital workers dealing with severe acute respiratory syndrome. Psychother Psychosom 75, 370-375. https://doi.org/10.1159/000095443

Mead, G.E., Morley, W., Cambell, P., Greig, C.A., Mc Murdo, M., Lawlor, DA. (2009). Exercise for depression. Cochrane Database Syst Rev, (3), CD004366.

Most, E.I., Scheltens, P., Van Someren, E.J. (2010). Prevention of depression and sleep disturbances in elderly with memory-problems by activation of biological clock with light-a randomized clinical trial. Trials, 11, 19. Pappa, S., Natella, V., Giannakas, T, Giannakoulis, V. G., Papoutsi, E., Katsaounou, P. (2020). Prevalence of depression, anxiety, and insomnia among healthcare workers during the COVID-19 pandemic: A systematic review and meta-analysis. Brain, Behavior and Immunity, 88, 901-907. https://doi.org/10.1016/j.bbi.2020.05.026

Paul, M.A., Gray, G.W., Lieberman, H.R., Love, and col. (2011). Phase advance with separate and combined melatonin and light treatment. Psychopharmacology, 214(2), 515-23.

Peduzzi, P., Concato, J., Kemper E, Holford TR, Feinstein AR. (1996). A simulation study of the number of events per variable in logistic regression analysis. J Clin Epidemiol, 49, 1373-1379.

Pfizer Inc. (2010). "Instrucciones para Cuestionario de Salud del Paciente (PHQ) y medidas de GAD-7”. Pfizer.

Postuma, R. B., Arnulf, I-, Hoglm, B., Iranzo, A., Miyamoto, T., Dauvilliers, Y. et al. (2012). A single-question screen for rapid eye movement sleep behavior disorder: A multicenter validation study. Mov Disord, 27, 913-916. http://dx.doi.org/10.1002/mds.25037.

RTM IV. (2012). Recomendaciones terapéuticas en los trastornos mentales. En P. Soler Insa, J. Gascón Barrachina (Edit.), Comité de Consenso de Catalunya en terapéutica de los trastornos mentales, 4ta ed., Cyesan.

Saxena, S. K. (2020). Coronavirus Disease 2019 (COVID-19) Epidemiology, Pathogenesis, Diagnosis, and Therapeutics. Medical Virology: from Pathogenesis to Disease Control. Springer. https://doi.org/10.1007/978981-15-4814-7

Soria, V., Urretavizcaya, M. (2009). Circadian rhythms in depresión. Actas Esp Psiquiatr, 37(4), 222-24.

Souetre, E., Salvati, E., Belugou, J.L. et al. (1988). Circadian rhytms in depresión and recovery: Evidence for blunted amplitude as the main chronobiological anormality. Psychiatry Reserch, 28, 263-78.

Spitzer, R. 1., Williams, J.B.W., Kroenke, K., Lowe, B. (2006). A brief measure for assessing Generalized Anxiety Disorder: The GAD-7. JAMA, 166, 1092-1097.

Terman, M., Terman, J.S. (2004). Light therapy for seasonal and non seasonal depression: efficacy, protocol, safety, and side effects. CNS Spectrums, 10, 647-63.

Vyas, N., Suno, E. (2020, 14 de agosto). National Sleep Foundation Recommends New Sleep Times. https:// www.sleepfoundation.org/press-release/ national-sleep-foundation-recommends-new-sleep-times/page/0/1 